\title{
Magnetic Resonance Imaging of a Primary Site with and without Contrast
}

National Cancer Institute

\section{Source}

National Cancer Institute. Magnetic Resonance Imaging of a Primary Site with and

without Contrast. NCl Thesaurus. Code C137902.

Magnetic resonance imaging of the primary site without a contrast agent to enhance the image. 